
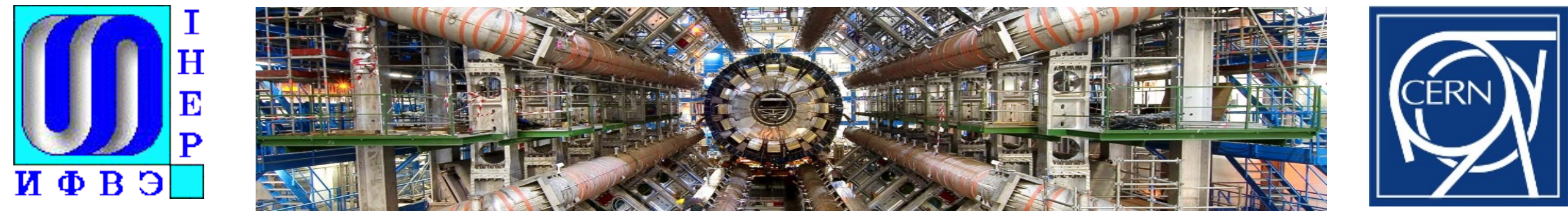

\title{
Upgrade of the ATLAS hadronic Tile Calorimeter for the High luminosity LHC
}

Alexander Solodkov

IHEP, Protvino \& CERN, Geneva on behalf of the

ATLAS Tile Calorimeter System

Instrumentation for Colliding Beam Physics February $27^{\text {th }}-$ March $3^{\text {rd }}, 2017$ Novosibirsk, Russia

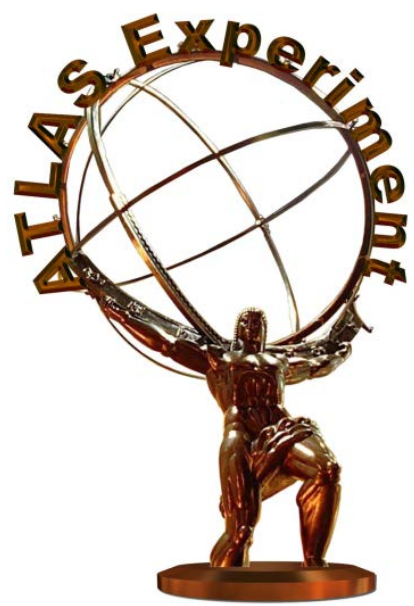




\section{Scope and motivation of the HL-LHC upgrade of Tile Calorimeter}

- The detector components (absorber, scintillating tiles, fibers, and PMTs) are largely in good shape and do not have to be replaced.

- The readout electronics has to be replaced:

1. The present digital readout is not compatible with the HL-LHC architecture.

2. Aging of parts (time \& radiation). Some parts are discontinued.

3. To provide full-granularity digital data to the Level-0/1 triggers at $40 \mathrm{MHz}$

- The present on-detector electronics is designed to output digital data at the maximum rate of about $100 \mathrm{kHz}$

- The digital data is stored on detector in a $2.5 \mu$ s-long pipeline.

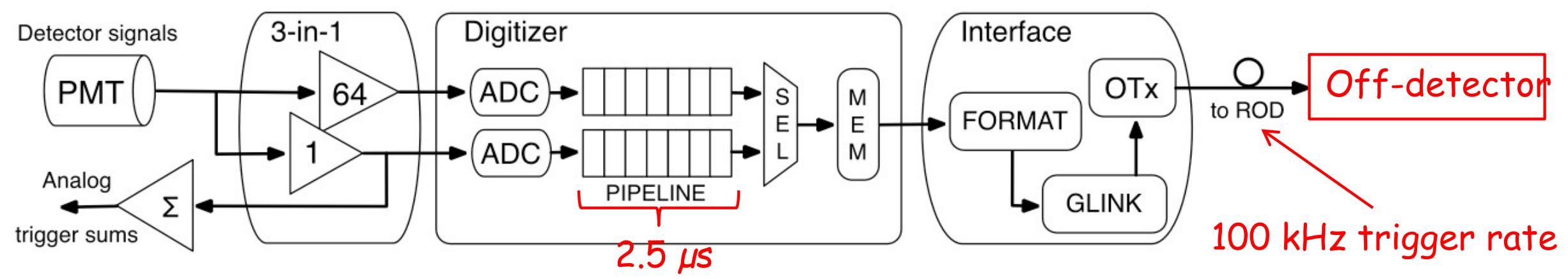

Without the upgrade digital readout of TileCal will not be possible in Phase II. 


\section{The new readout architecture}

- The main difference between the present and the upgrade readout architectures is that all digital data is transmitted off-detector at $40 \mathrm{MHz}$.

- The data is pipelined and processed in the off-detector pre-processor boards (TilePPrs).

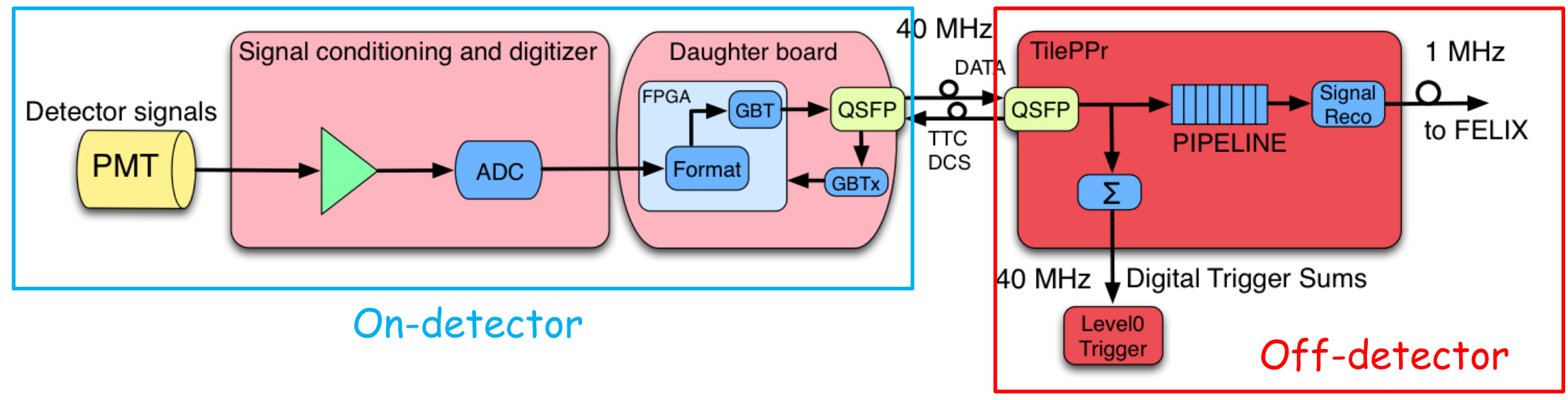

- The new on-detector electronics is much simpler and does not have any readout limitations like the present system.

- The upgrade also improves flexibility of the off-detector data processing for the Level0/Level1 triggers.

- Redundancy and reliability are the key themes for the upgrade (data transmissions and power distribution) 


\section{Digitization of the PMT signals}

- Processing of PMT pulses is a major challenge because the pulses are short and subject to photo-statistical fluctuations.

- For each pulse we need to obtain time and total charge. The total charge is proportional to the number of photo-electrons and, therefore, to the energy deposited in the corresponding TileCal cell.

- We have developed three frontend technologies to process PMT signals:

1. “3-in-1"

2. FATALIC

3. QIE

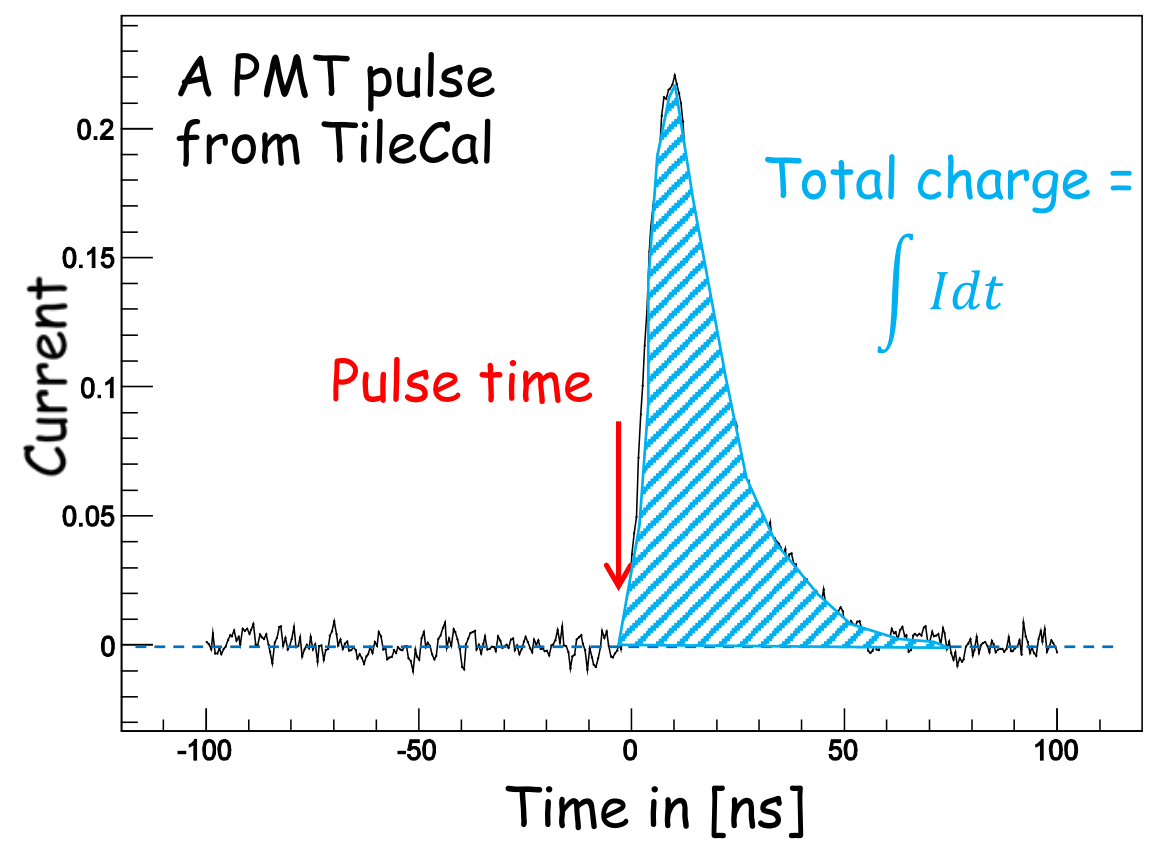




\section{1: The 3-in-1 front-end signal processing}

- The 3-in-1 signal processing approach uses a shaper circuit to transform a PMT pulse into an easy to digitize waveform/pulse whose amplitude is proportional to the total charge of the original PMT pulse.
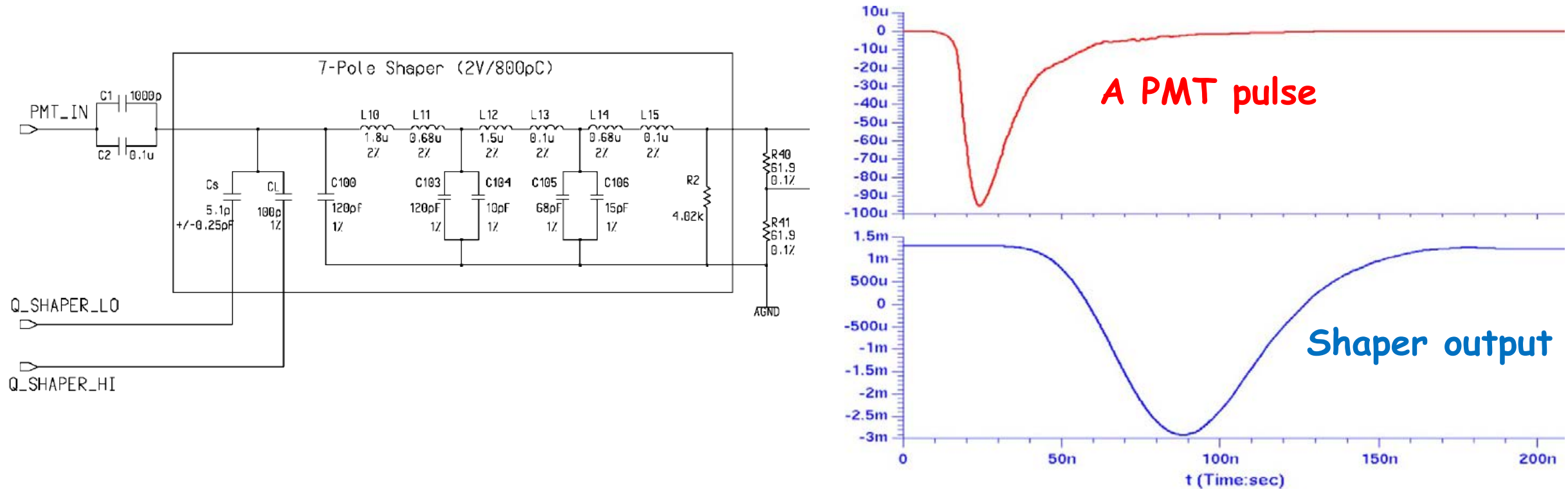

- Actually it is an optimized version of the current 3-in-1 card

- The shaper circuit contains only passive elements and its performance is very predictable and well-characterized with calibration procedures. 


\section{1: The 3-in-1 front-end signal processing}

- The 3-in-1 circuits are built of discrete components.

- The analog signals are processed in the front-end cards connected to the PMTs.

- Shaped pulses from 12 front-end cards are digitized on a main board.

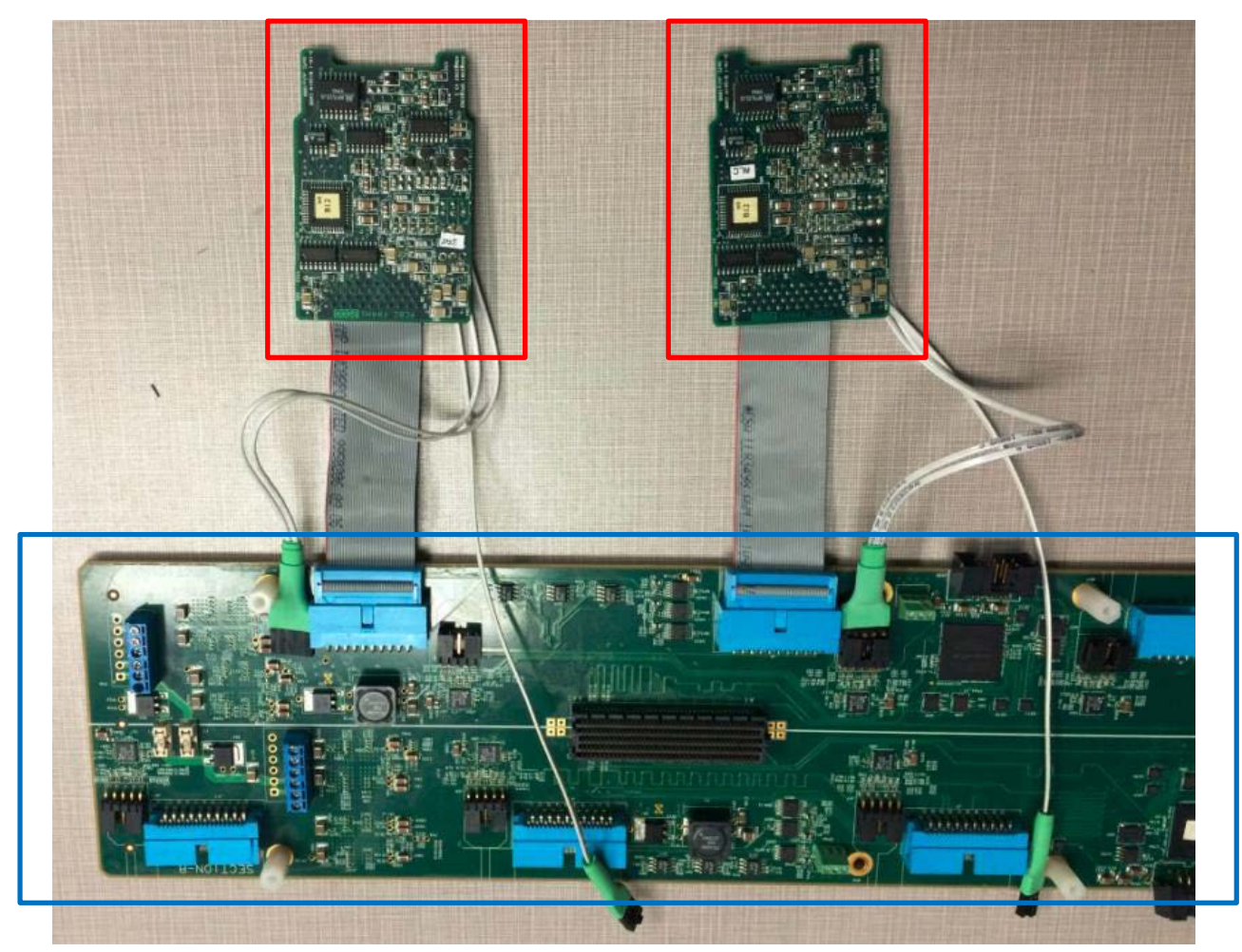

- The upgrade brings

- Better digitization: 12bits ADCs instead of 10 bit

- Modern, better performing components

- Even lower electronic noise than the present system.

- The new 3-in-1 system is compatible with the analog trigger and can be installed into TileCal before the HLLHC upgrade 


\section{2: The FATALIC signal processing}

- A new technology for TileCal.

- Implemented as an application-specific integrated circuit (ASIC).

- $\quad$ FATALIC $=$ Front-end ATLas TIle Circuit

- The circuitry is different from 3-in-1.

- The FATALIC approach also uses shaping like the 3 -in- 1 but the pulse shape is different. Digitization is done inside the chip.

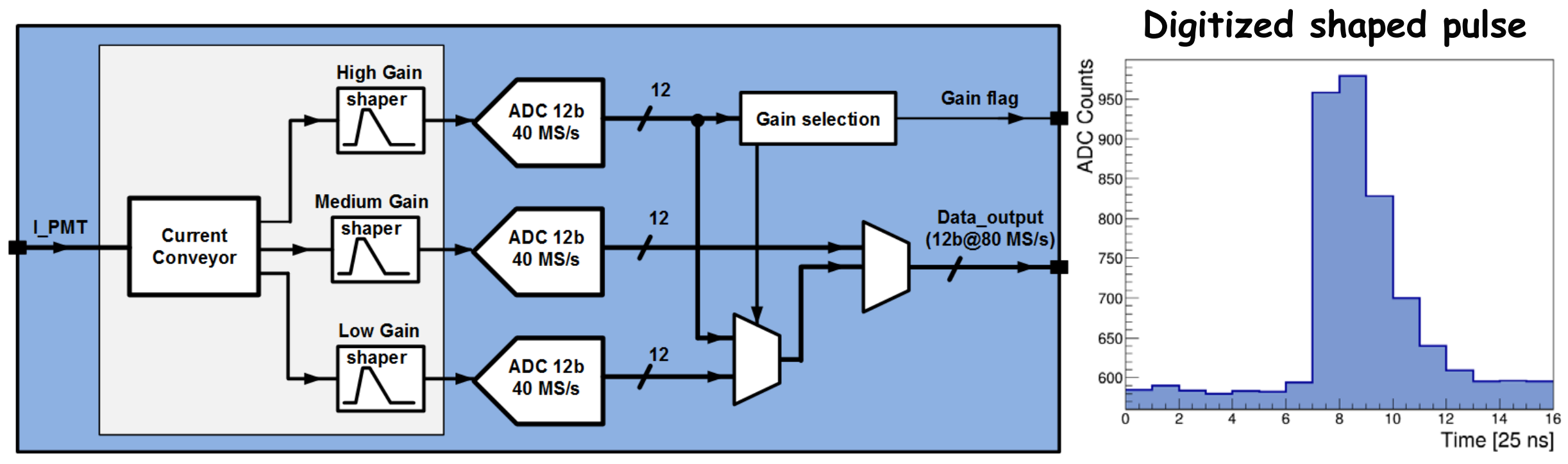




\section{2: The FATALIC signal processing}

- Several versions of FATALIC have been produced \& tested already

- After 2016 testbeams the FATALIC_4 was revised to improve:

1. Integrity of the digital output

2. Linearity across the entire dynamic range ( $1 \%$ up to $1.2 \mathrm{nC}$ )

3. Calibrations with a Cs source.

- Simulated performance for FATALIC_5 is within specifications.

- FATALIC_5 was sent to the foundry in February 2017.

- New chips should be ready in May-July 2017 for testbeam evaluation.

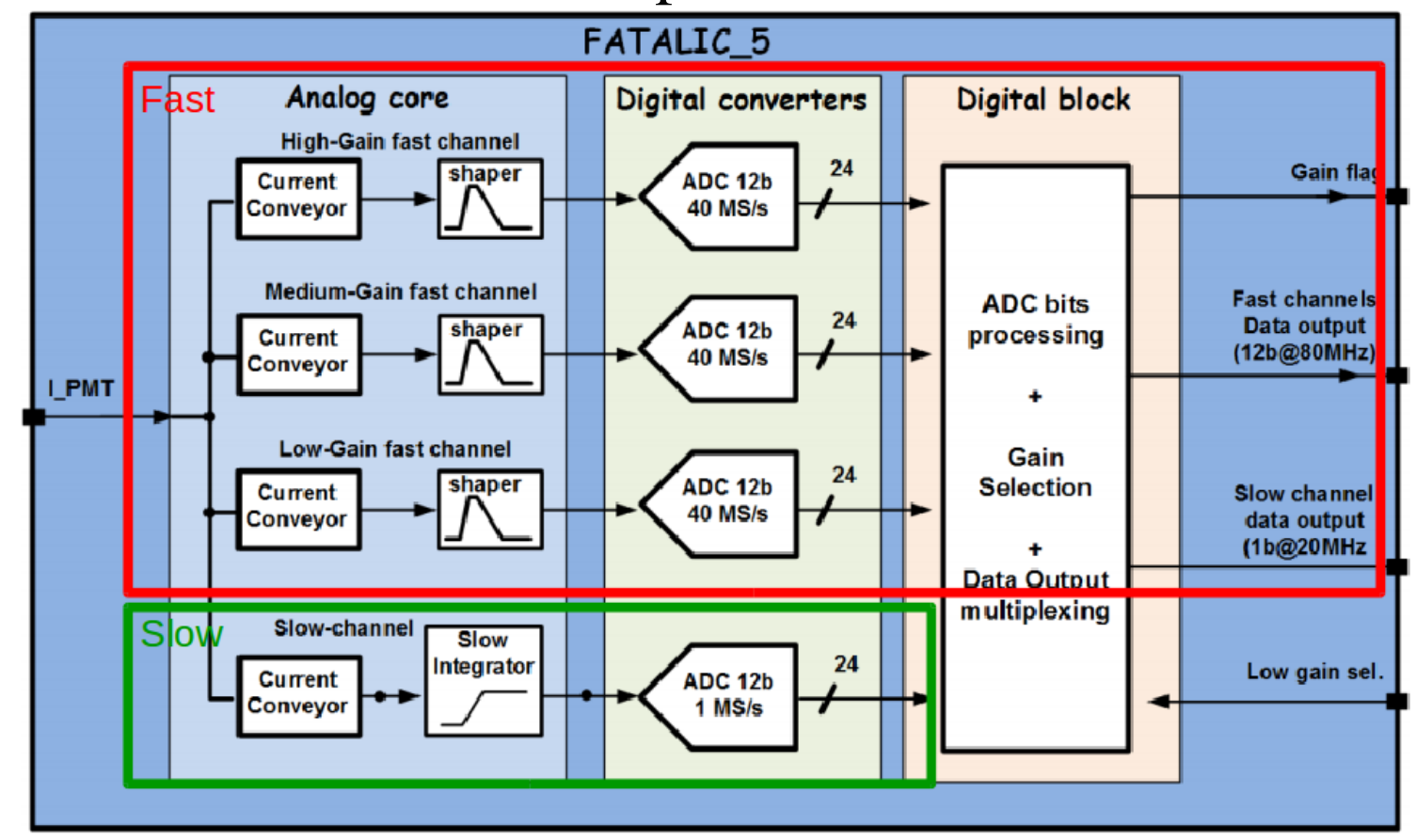




\section{3: The QIE signal processing}

- QIE12 is TileCal tailored QIE family ASIC, used largely by CMS

- The QIE circuit does not shape the PMT pulse to digitize at 40 Msps. Instead, it directly integrates the PMT anode current in 25 ns intervals.

- Every 25 ns a QIE gives two numbers:

- Charge (current integrated over a 25 ns period).

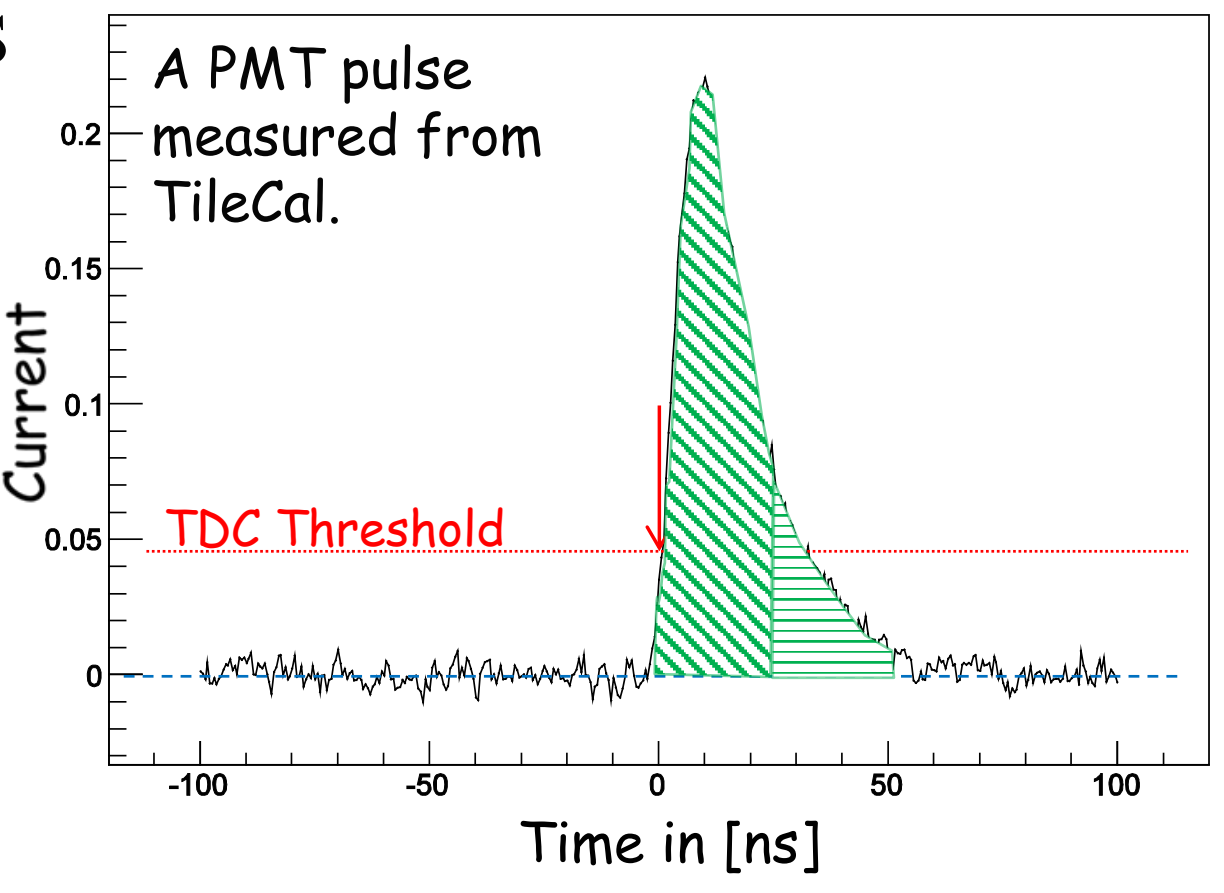

- Time (when current crossed a threshold). 1 ns resolution

- The total charge of a PMT pulse (energy) is obtained as a sum of two or three QIE samples

- This approach has been used in many experiments (CMS, CDF, etc). 


\section{3: The QIE signal processing}

- The PMT current is integrated in a bank of capacitors.

- The capacitors are time-multiplexed for deadtimeless operation at $40 \mathrm{MHz}$.

- The current splitter is used to achieve the required dynamic range ( $>17$ bits).

- The QIE12 ASIC is designed to be radiation tolerant and tailored for TileCal.

- All the signal processing (integration and digitization) is done in the front-end boards.

- QIE main boards have mostly point-to-point signal connections and power distribution.
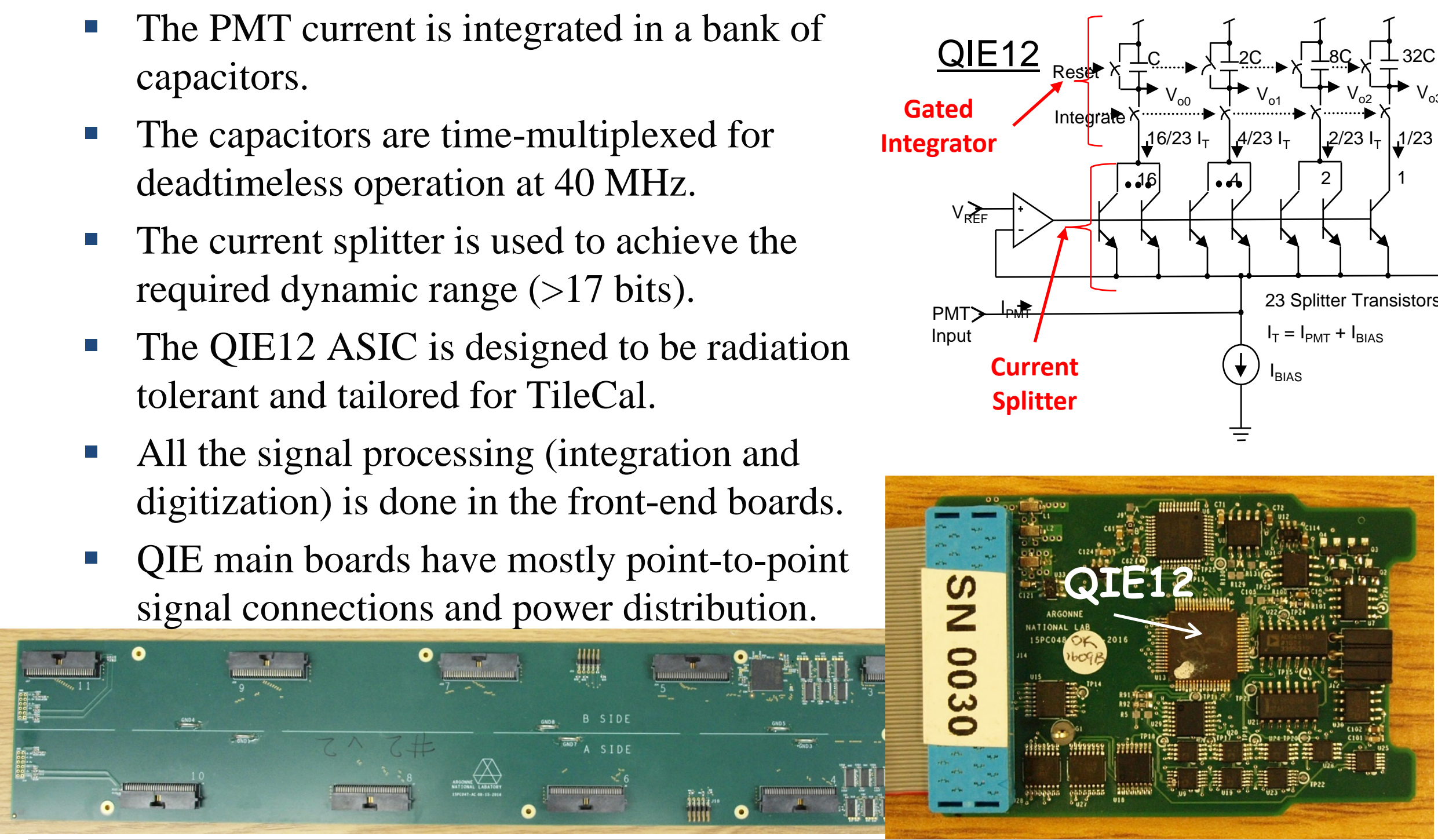


\section{Cherenkov}

counters

Separate $\mathrm{p} / \pi / \mathrm{e}$ for

$\mathrm{E}($ beam) $\leq 50 \mathrm{GeV}$

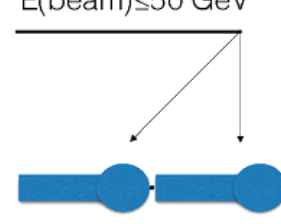

2 Trigger

scintillators
2 wire chambers

Measure beam

impact point on TileCal

\section{Testbeam}

\section{measurements}

- 3 TileCal modules equipped with Phase-II upgrade electronics together with modules equipped with the legacy system were exposed to different particles and energies in three testbeam

campaigns during 2015 and 2016.

- Half-module (LBC65) has been equipped with 3-in-1 front-end option

- Another half-module (LBA65) has been instrumented with other options QIE and FATALIC.

positive eta

Muon

hodoscope negative eta

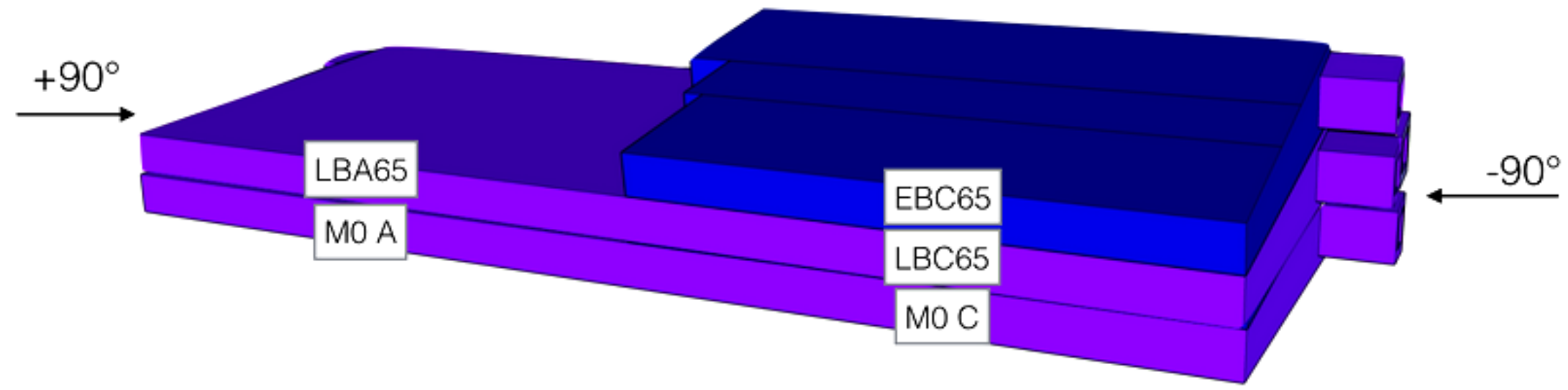




\section{Testbeam measurements}

- Overall, good performance for 3-in-1 and QIE systems have been demonstrated

- Agreement among all options in terms of energy calibration (using $100 \mathrm{GeV}$ electrons)

- Signal to noise ratio is improved with respect to legacy system (factor of 2)

- Muon signal is very well visible

- FATALIC option showed higher noise and will be improved this year

Muon signal in smallest cell @ 200

Correlation of 2 PMTs of the same cell
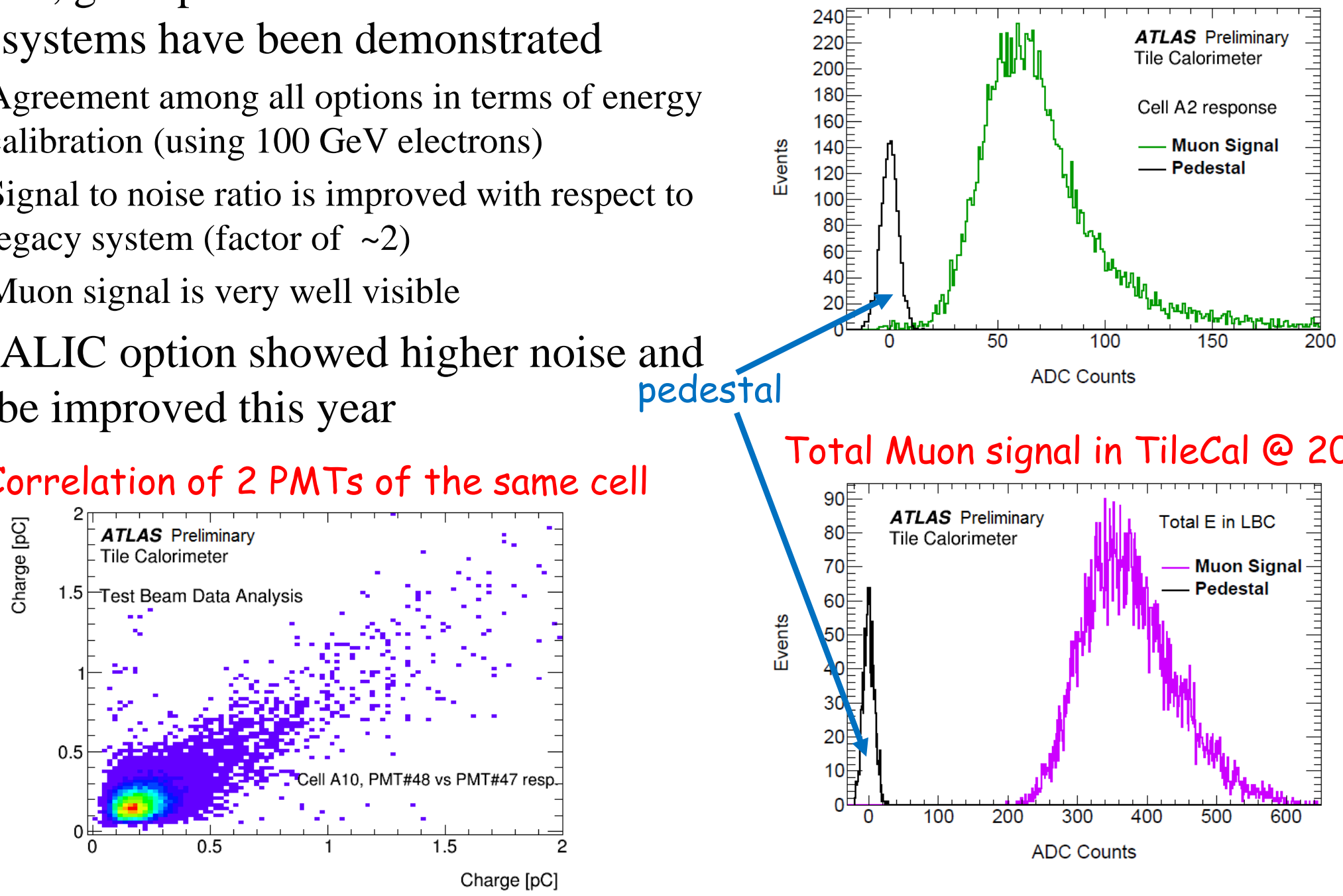

Total Muon signal in TileCal @ 20。 


\section{The digital data path}

- Digital data from 12 front-end readout channels (a main board) is received by a daughter board. The daughter board serializes the data and transmits it via multiple 9.6 Gbps fiber-optic links for redundancy

- PreProcessor (TilePPr) receives data from multiple daughter boards to pipeline it and to prepare the data for the trigger system.

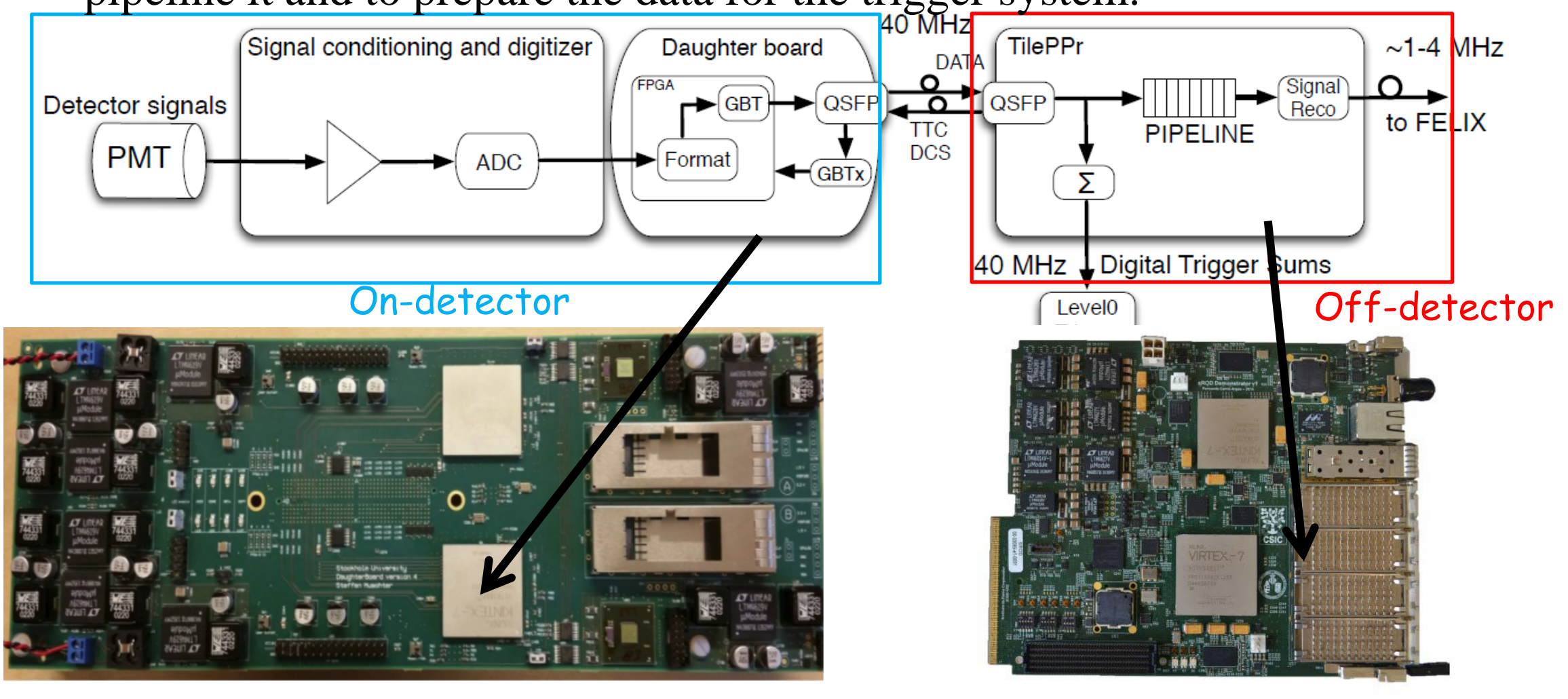




\section{Off-detector electronics}

- TilePPr demonstrator (used at the testbeam)

- Designed in an Advance Mezzanine Card (AMC) format which can be operated in an ATCA or standalone framework.

- Upon the reception of a trigger signal, the data is formatted and transmitted

- to the legacy Read-Out Driver (ROD) for backward compatibility with the current system

- to the Front-End Link eXchange (FELIX) for full precision readout

- TilePPr for HL-LHC upgrade

- Will be implemented in a full size ATCA blade format

- In addition to normal readout will provide also preprocessed information to Level0/Level1 trigger

- 32 TilePPr will be needed to readout whole TileCal

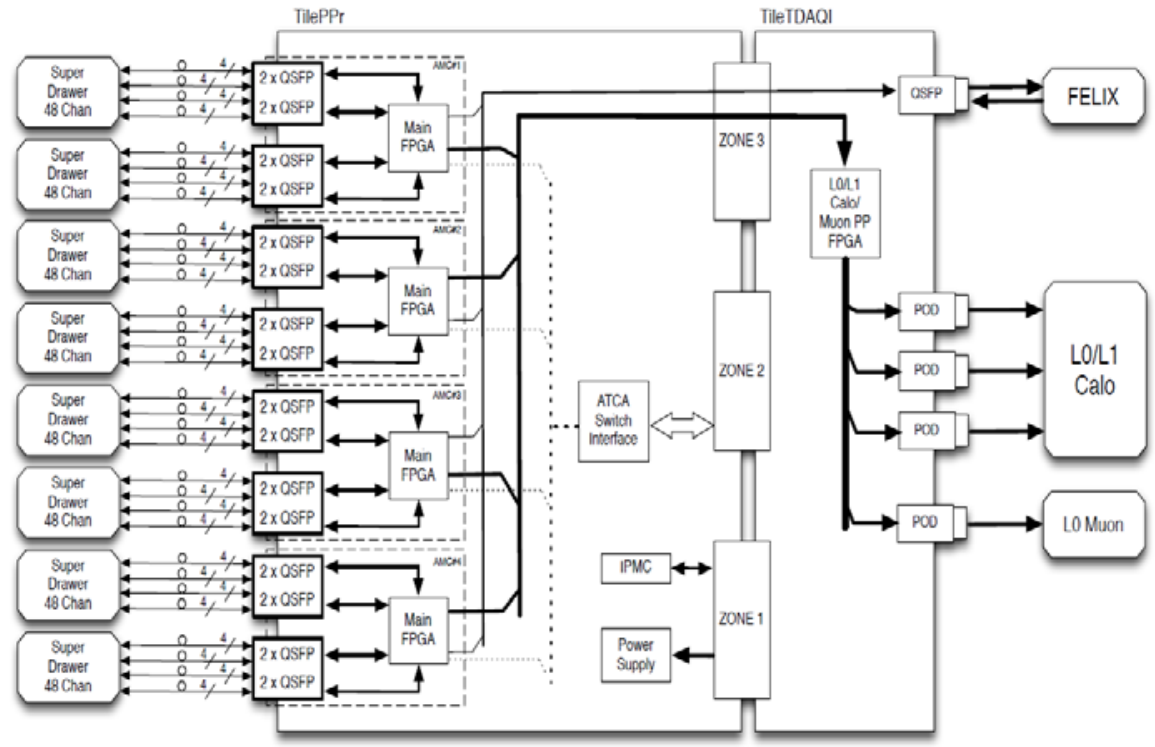




\section{Low voltage power supplies}

- The power distribution scheme is revised for the HL-LHC upgrade.

- Redundancy is increased.

- The power supplies produce $+10 \mathrm{~V}$ and the other voltages are derived in situ on the main boards and daughter boards.

- Used for test beam evaluation.

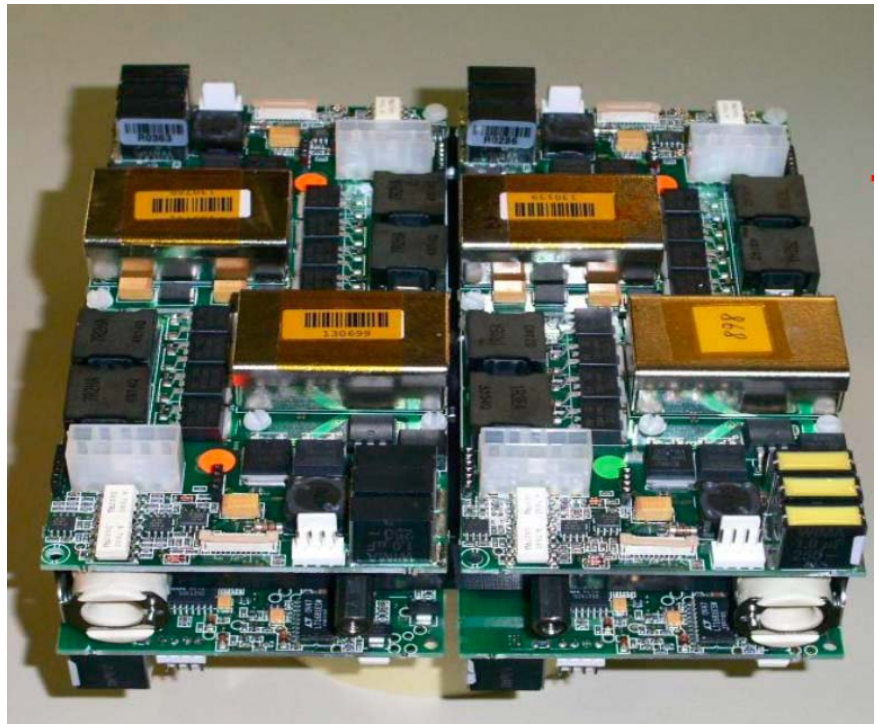

flvPs Power Connectivity Block Diagram
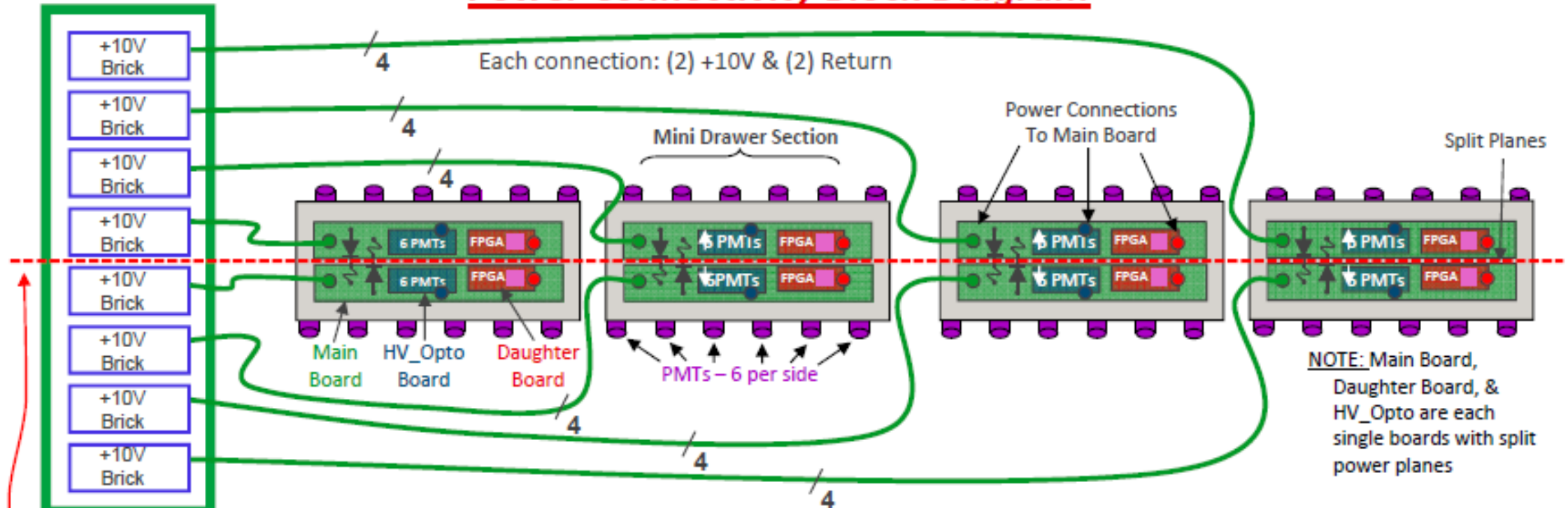

Redundancy Line

INSTR 2017, BINP, Novosibirsk, Russia, 27-Feb - 03-Mar 2017 


\section{HV distribution systems}

- Two systems have been designed for distributing high voltage to the PMTs.

- HV_opto uses existing cables to derive the voltages specific for each PMT on detector.

- Small number of channels are subject to 1 SEU per year. All the safety factors are included. The SEU's are correctable.
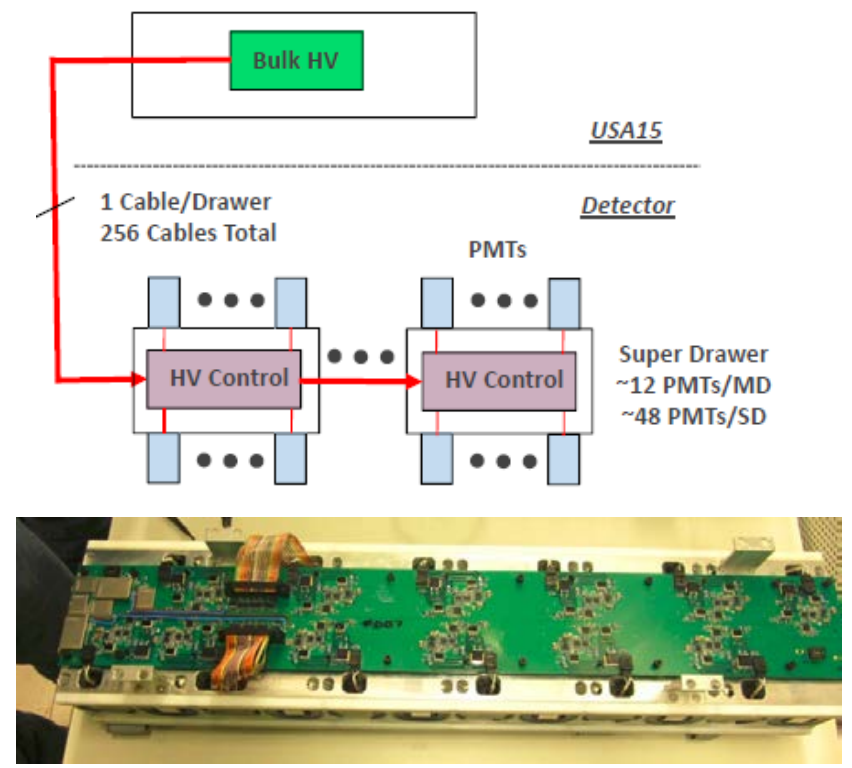

- HV_remote requires new 1024 120m-long 12-channel cables.

- Radiation is not an issue.

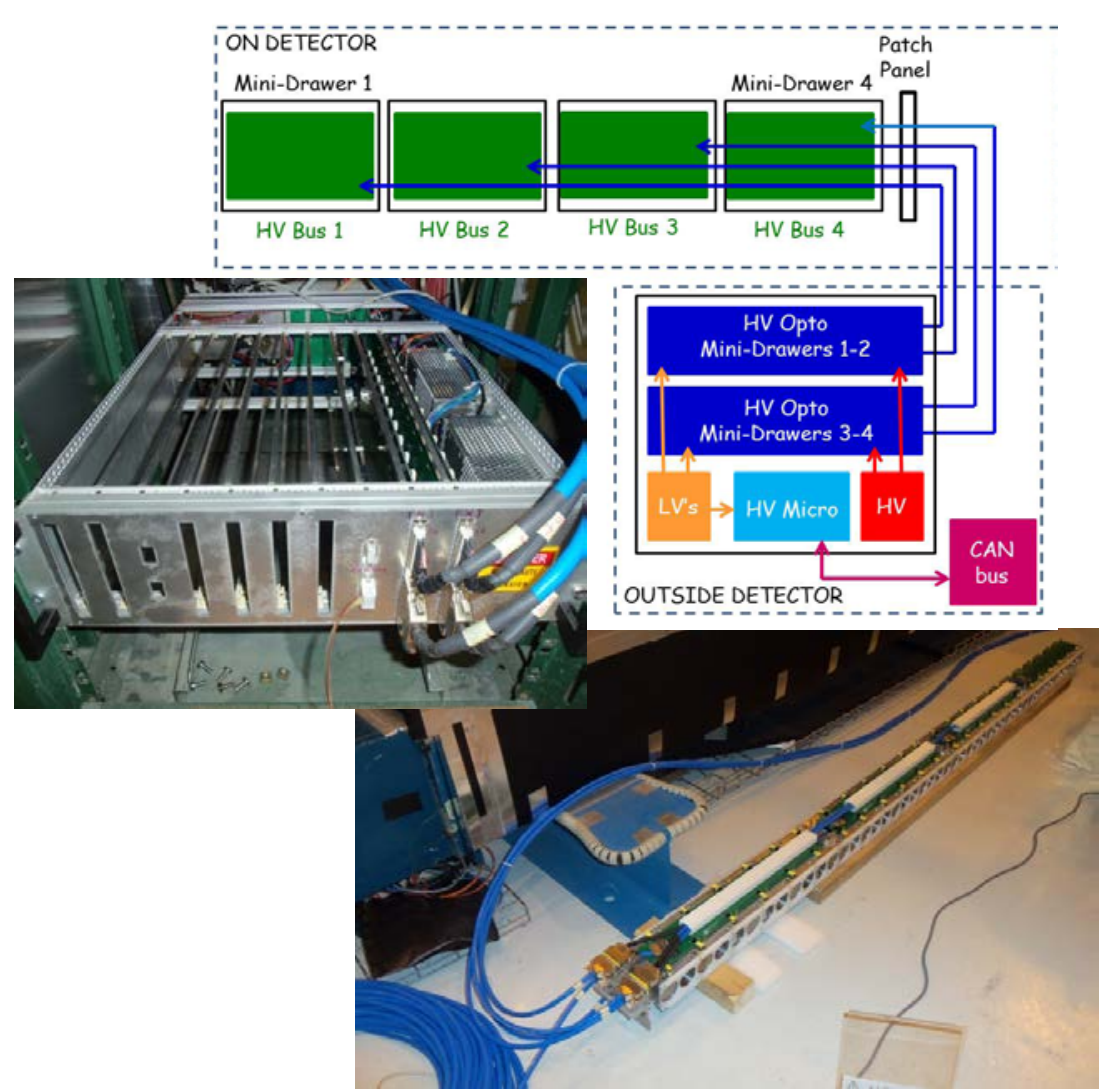

- The main difference between the two options: regulation on- and off-detector. 


\section{High Granularity Upgrade of TileCal readout}

- The promising high-granularity (MA-PMT) readout option have been proposed recently

- The goal is to increase the spatial resolution for the detector by

- Increasing A layer Z (eta) granularity by a factor of 4

- Increasing BC layer longitudinal granularity by a factor of 2

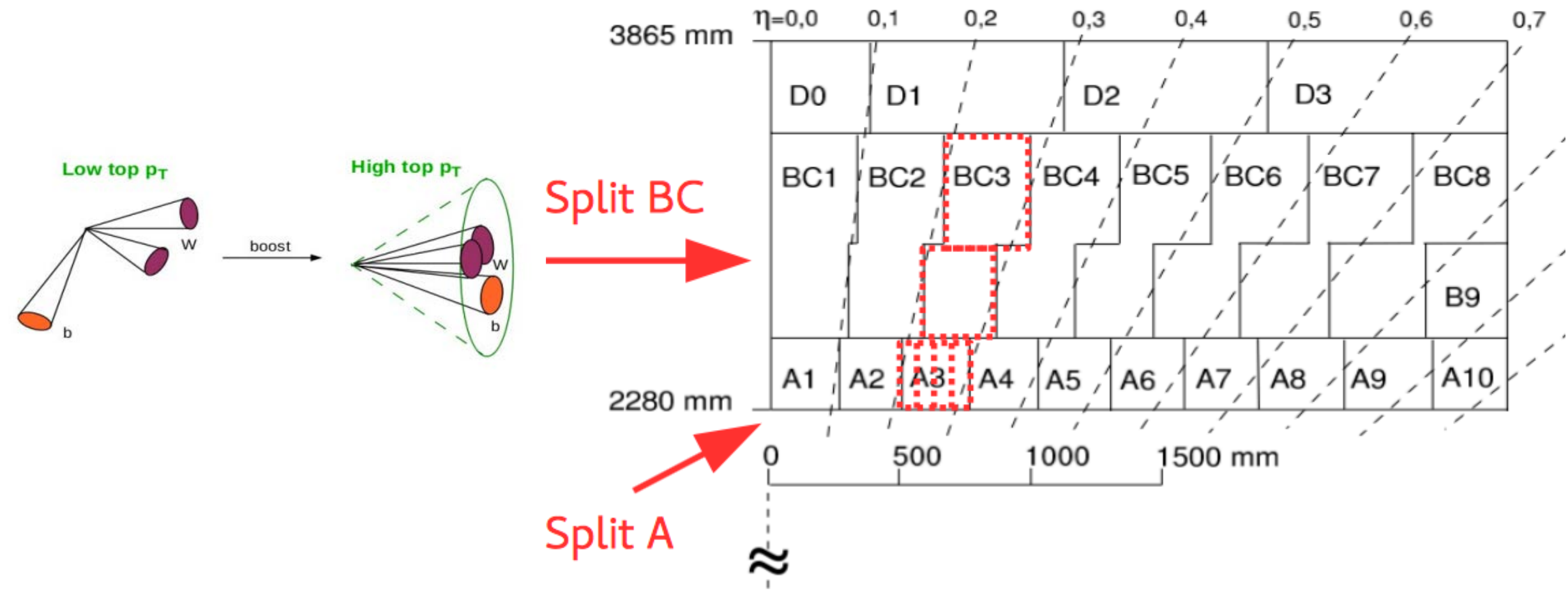




\section{High Granularity Upgrade of TileCal readout}

- The time and effort required to mature this option is not easily compatible with the current tight schedule of ATLAS Phase-II upgrade.

- We will finalize the feasibility studies in simulation, optics and electronics, and try to understand how this option can be possibly implemented in future upgrades, and that the existing design revisions and modifications can be compatible with the possible later upgrade to the MA-PMT, without large changes in the interface to the services to the detector and DAQ.

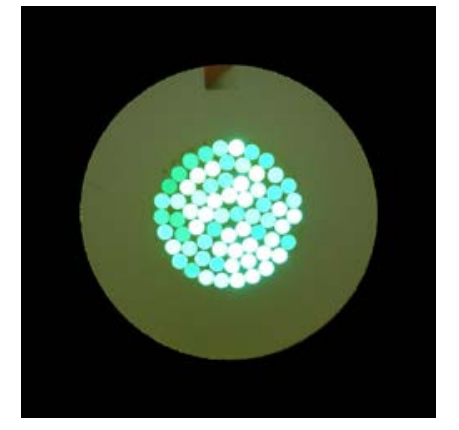

Fiber diameter - $1 \mathrm{~mm}$ From 30 to 70 fibers in bundle
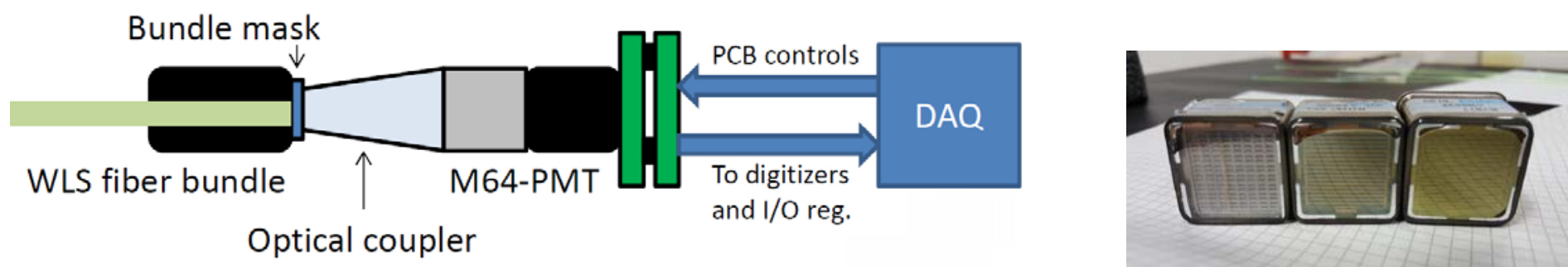

Hamamatsu R7600-300-M64 8×8 MA-PMT Pixel size $2.35 \mathrm{~mm} \times 2.35 \mathrm{~mm}$ 


\section{Conclusions and Outlook}

- The upgrade architecture is mature and well understood.

- “3-in-1" and QIE front-end technologies were successfully evaluated at the testbeam and do not require any major revisions.

- FATALIC_5 is expected later this year.

- The digital data path and detector control worked well.

- All the results are documented in the Initial Design Report

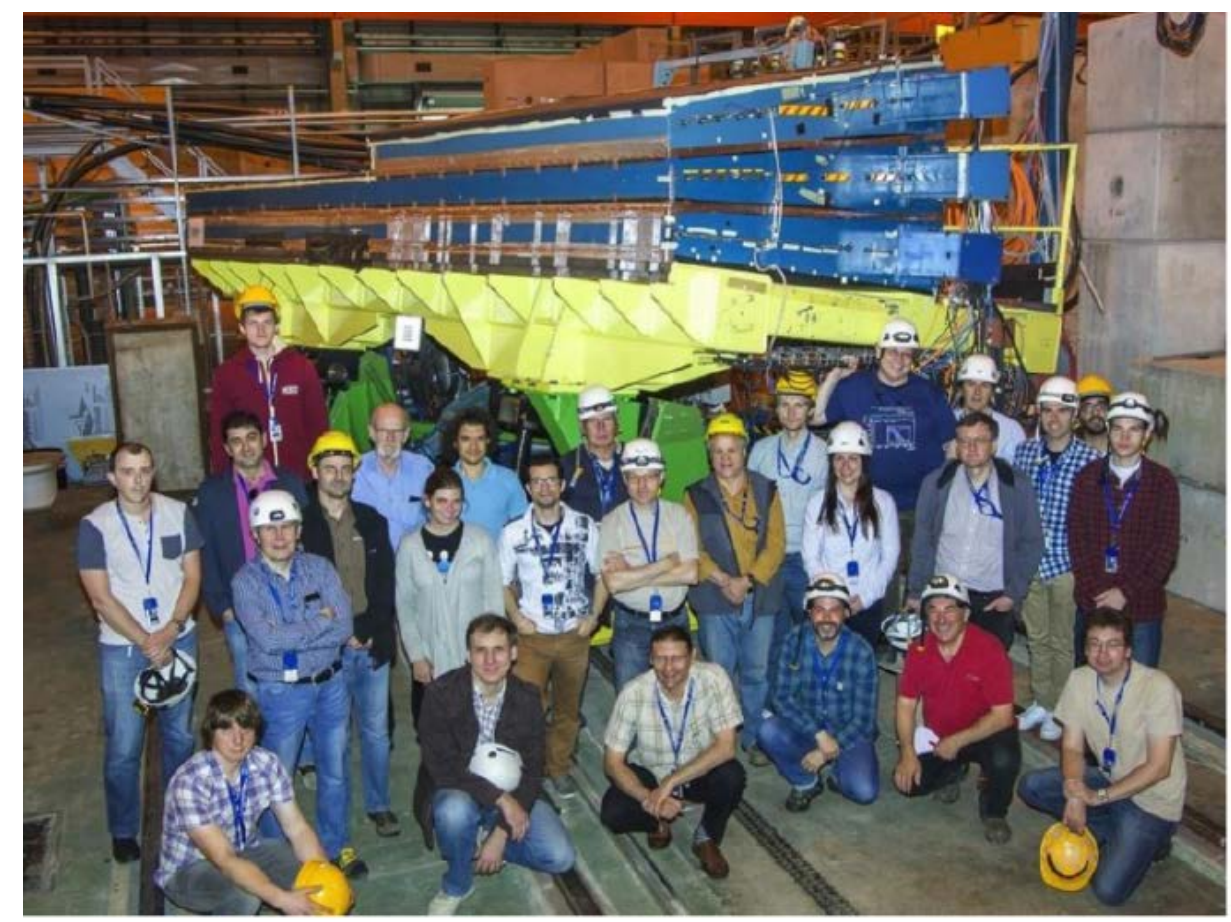

- The FE and HV option final selection process have been defined based on extensive comparison of the performances and other criteria. We plan to complete the decision by the end of summer.

- Technical Design Report is planned for the end of 2017

- MA-PMT option is a good candidate for TileCal upgrades beyond Phase-II 


\section{BACKUP}




\section{Tile Calorimeter - central hadronic}

calorimeter in ATLAS

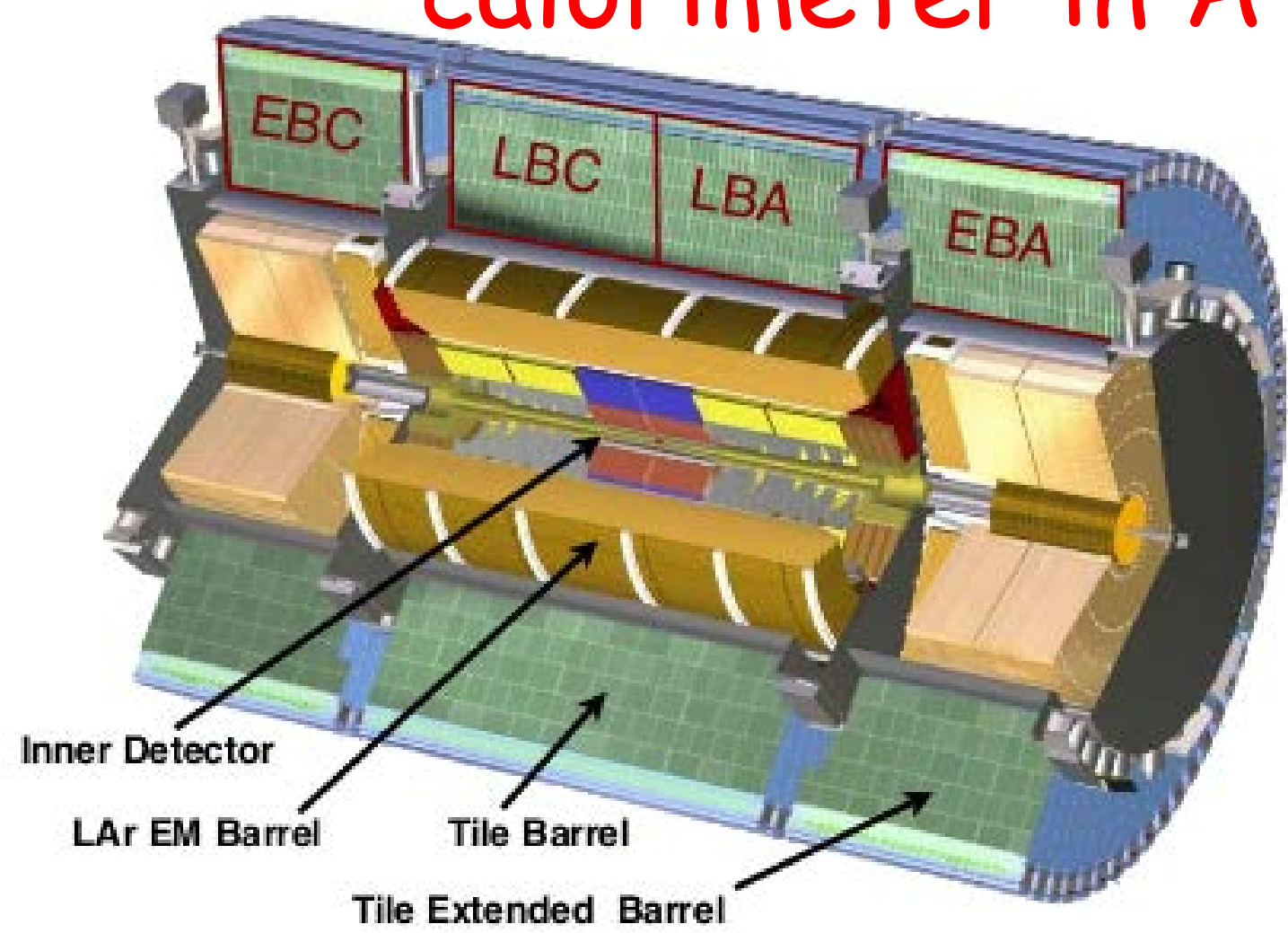

Dimensions

- Diameter: $8.5 \mathrm{~m}$

- Length = $12 \mathrm{~m}$

- Weight: $2900 \mathrm{~T}$

- Hadronic sampling calorimeter: iron/scintillator 5:1

- 3 cylinders with coverage: $|\eta|<1.0$ in barrel, $0.8<|\eta|<1.7$ in extended barrel

- 64 independent modules in every cylinder

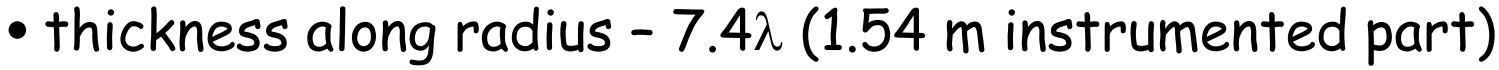

- Aim for jet energy resolution: $\frac{\Delta E}{E} \sim \frac{50 \%}{\sqrt{E}} \oplus 3 \%$ 


\section{TileCal readout}
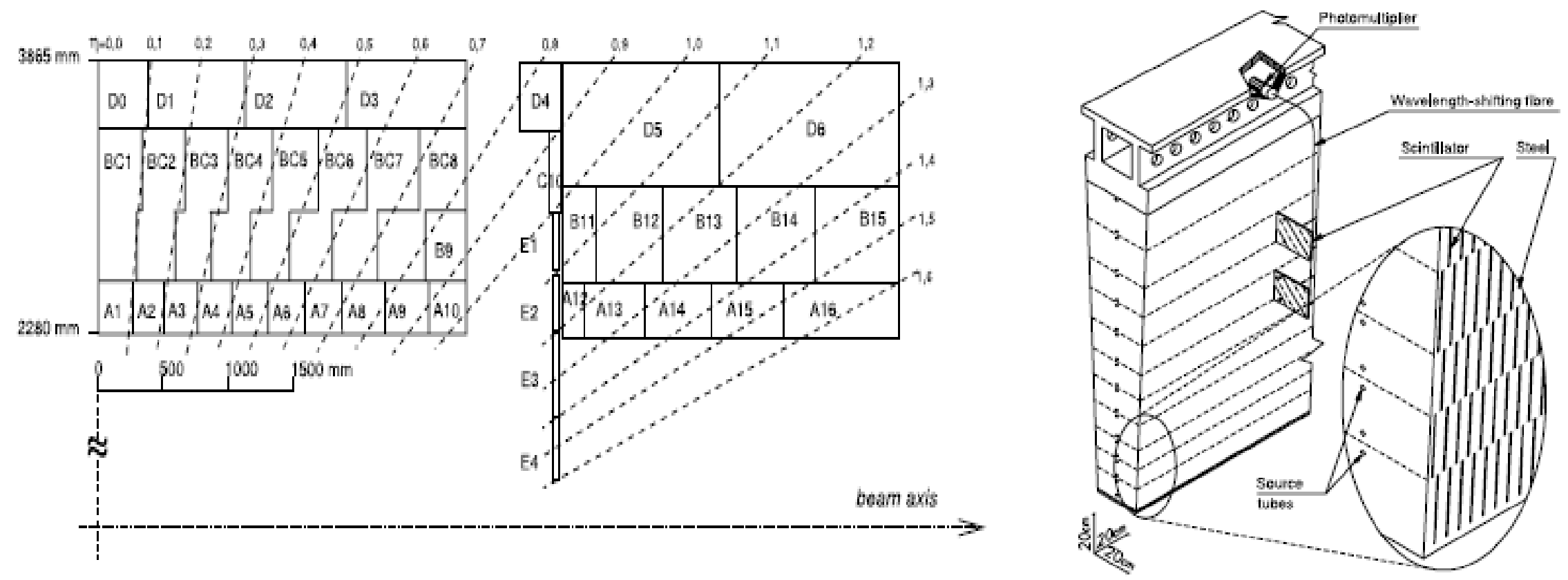

- Every scintillating tile in 11 rows is read out by 2 wavelength shifting fibers

- Fibers go along both sides of every module to outer radius and are grouped together into pseudo-projective geometry cells in 3 layers

- Granularity $\Delta \eta \times \Delta \phi=0.1 \times 0.1$ in first two and $0.2 \times 0.1$ in outermost layer

- number of tiles in one cell varies from 16 to 300

- 5182 cells, 9852 channels in total (double readout for normal cells, single readout for special cells E1-E4)

- Readout is organized in four partitions

- barrel is split in two partitions called LBA for $\eta>0, L B C$ for $\eta<0$

- two extended barrels called EBA and EBC 


\section{A bit of history....}

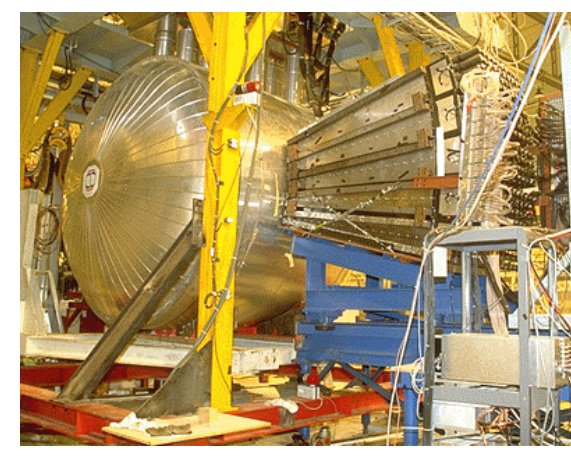

1993-1995 R\&D

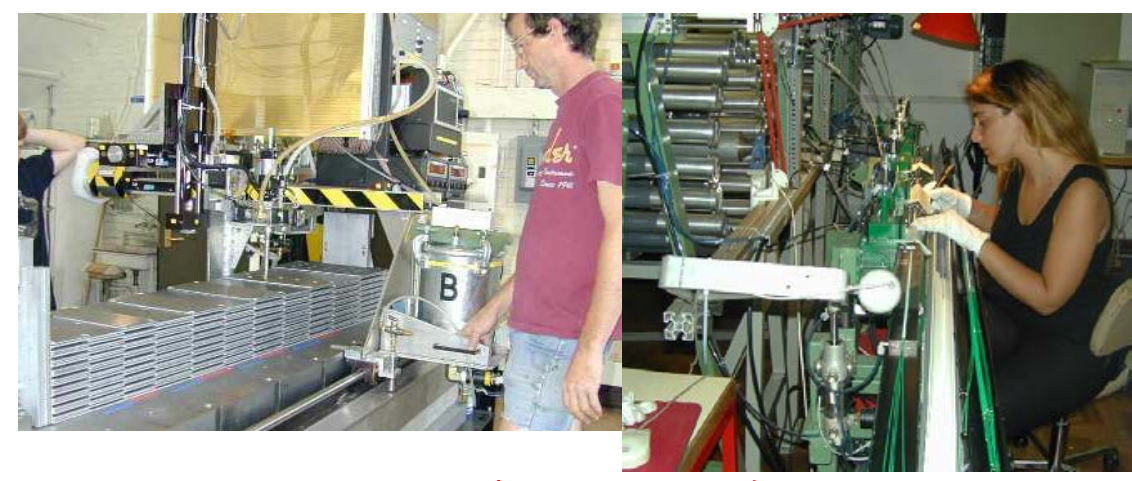

1996-2002 Mechanics and optics construction

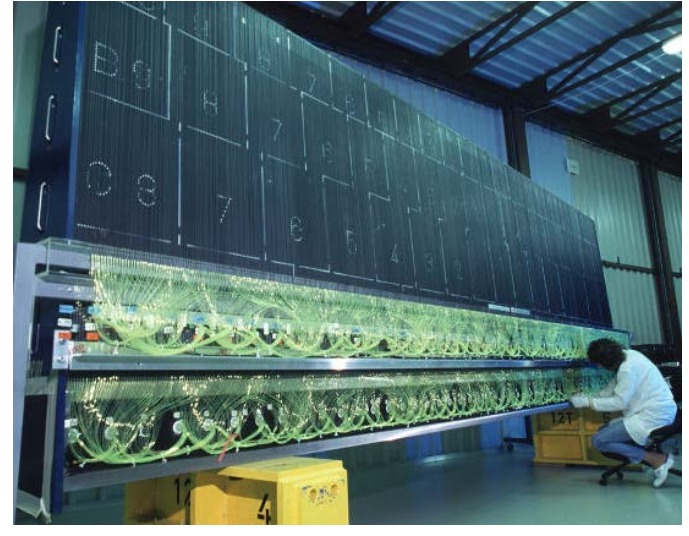

1999-2002

Instrumentation

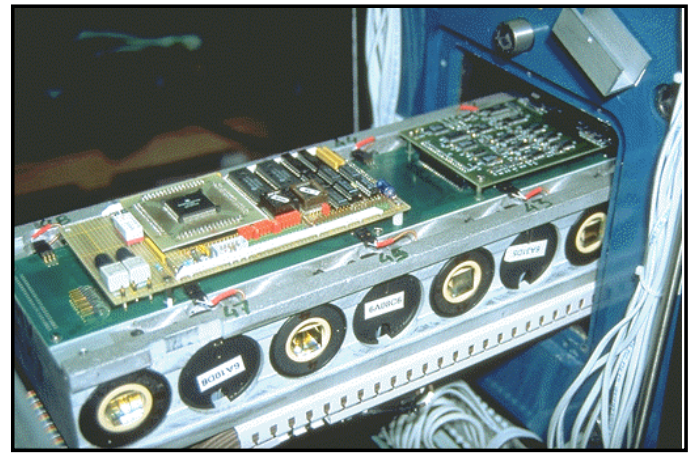

1999-2004: Electronics construction

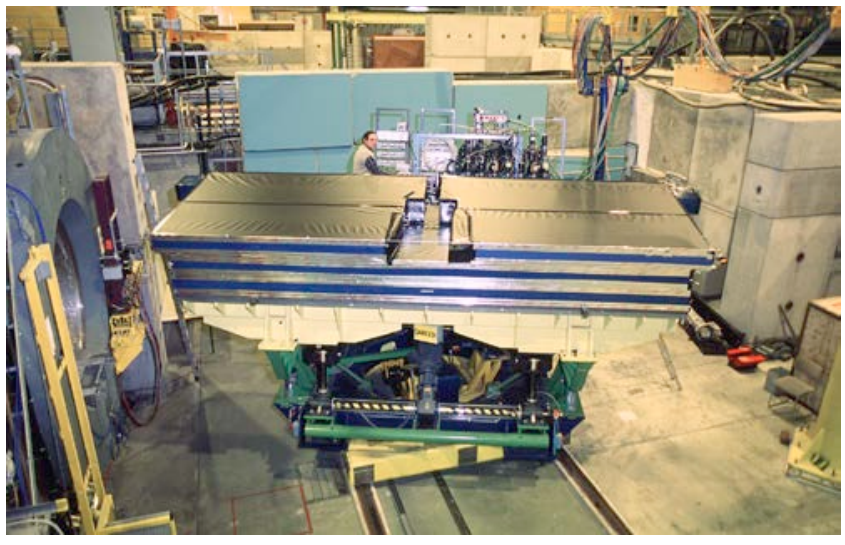

2001-2004: Calibration at the testbeam

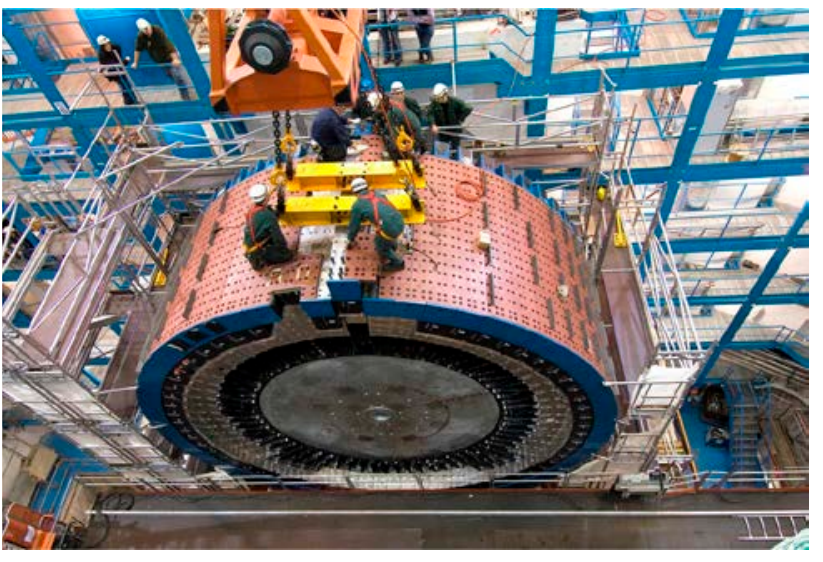

2004-2006: Installation

Since 2006 - commissioning using cosmic muons and calibration triggers Since 2009 - continuous operation in pp collisions at 7,8 and $13 \mathrm{TeV}$ 